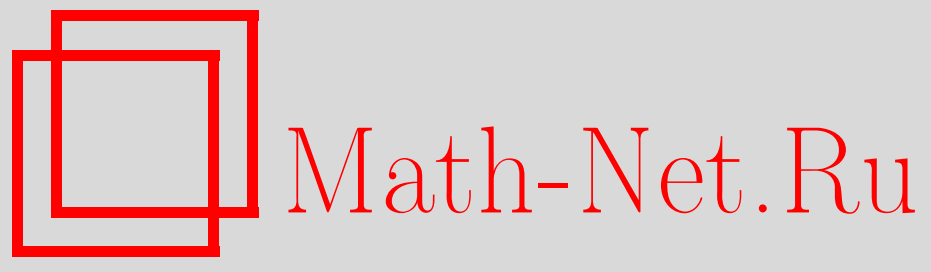

А. Д. Коршунов, Некоторые нерешенные задачи дискретной математики и математической кибернетики, УМH, 2009, том 64, выпуск 5, 3-20

DOI: https://doi.org/10.4213/rm9322

Использование Общероссийского математического портала Math-Net.Ru подразумевает, что вы прочитали и согласны с пользовательским соглашением http://www . mathnet.ru/rus/agreement

Параметры загрузки:

IP : 54.224 .187 .69

26 апреля 2023 г., 16:22:21

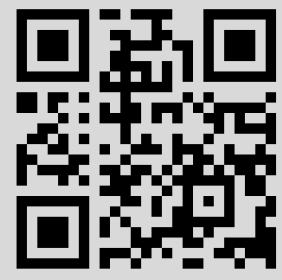




\title{
Некоторые нерешенные задачи дискретной математики и математической кибернетики
}

\begin{abstract}
А. Д. Коршунов
В области дискретной математики и математической кибернетики известно большое число нерешенных задач. Написание достаточно полного обзора по таким задачам связано с преодолением больших трудностей. Во-первых, спектр таких задач весьма широк и разнообразен. Во-вторых, степень трудности решения разных задач сильно отличаются друг от друга. Поэтому из множества задач даже в подробный и обстоятельный обзор следует включать не все задачи, а наиболее важные и существенные. Объективный отбор таких задач затруднителен.

В настоящую статью включены 13 нерешенных задач, относящихся к комбинаторной математике и теории сложности вычислений. Отобранные задачи отражают 50-летние исследования автора и по этой причине в определенной степени являются субъективными. Вместе с тем, эти задачи трудны и представляют значительный интерес для дискретной математики и математической кибернетики.

Библиография: 73 названия.

Ключевые слова: восстановление графа по подграфам, гамильтоновы циклы, дизъюнктивные нормальные формы, змея в булевом кубе, изоморфизм графов, нижние оценки, NP-полнота, полиномиальные задачи, протыкание кубов, сложно вычислимые булевы функции, совершенные двоичные коды, тройки Штейнера.
\end{abstract}

СОДЕРЖАНИЕ

1. NР-полные задачи . . . . . . . . . . . . . . . . . . . . . . . . . . . . . 4

2. Изоморфизм неориентированных графов $\ldots \ldots \ldots \ldots \ldots \ldots \ldots \ldots \ldots \ldots \ldots$

3. Нижние оценки сложности схем, вычисляющих индивидуальные булевы

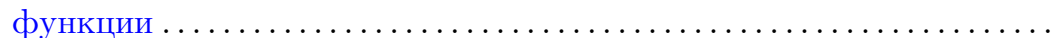

4. Сложность дизъюнктивных нормальных форм большинства булевых функций . . . . . . . . . . . . . . . . . . . . . . . . . .

5. Сложность покрытий числовых множеств арифметическими прогресси-

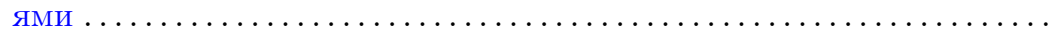

Работа выполнена при поддержке Российского фонда фундаментальных исследований (грант № 08-01-00611) и Программы фундаментальных исследований ОМН РАН “Алгебраические и комбинаторные методы математической кибернетики" (проект "Новые методы дискретной и комбинаторной оптимизации").

(C) А. Д. Коршунов, 2009 
6. Протыкание подкубов $n$-мерного булева куба................... 9

7. Длина максимальной змеи в $n$-мерном булевом кубе ............ 10

8. Гамильтоновы циклы в $n$-мерном булевом кубе.................. 11

9. Проблема гамильтоновости двух средних слоев $n$-мерного куба...... 12

10. Совершенные двоичные $(n, 3)$-коды $\ldots \ldots \ldots \ldots \ldots \ldots \ldots \ldots \ldots \ldots \ldots \ldots . . \ldots \ldots \ldots \ldots \ldots$

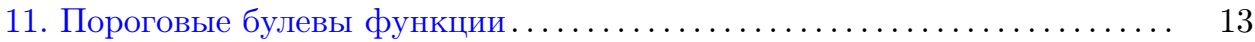

12. Число систем троек Штейнера ............................. 14

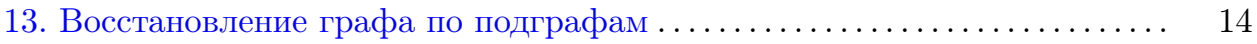

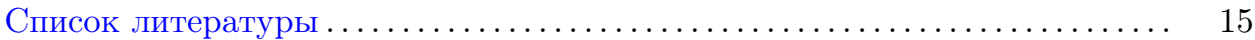

\section{NP-полные задачи}

Задача называется полиномиальной, если она может быть решена на одноленточной детерминированной машине Тьюринга с временно́й сложностью, не превосходящей $p(n)$, где $n$ - объем входных данных задачи, а $p(n)$ - подходящий полином от $n$. Класс полиномиальных задач обозначается через $\mathrm{P}$.

Задача называется NP-трудной, если существует одноленточная недетерминированная машина Тьюринга, на которой задача может быть решена с полиномиальной временно́й сложностью относительно объема входных данных задачи. Класс всех NP-трудных задач обозначается через NP.

Говорят, что задача $L_{1}$ полиномиально сводится к задаче $L_{2}$, если существует метод решения задачи $L_{2}$, который за полиномиальное время (относительно объема входных данных задачи $L_{1}$ ) можно преобразовать в метод решения задачи $L_{1}$.

Задача из NP называется NP-полной (универсальной), если к ней полиномиально сводится любая задача из NP.

Эти и некоторые другие понятия, связанные с NP-полнотой, впервые ввел С.А. Кук в элегантной работе [1]. В ней был заложен фундамент теории NP-полноты и доказано, что одна конкретная задача из класса NP, называемая задачей о выполнимости, является NP-полной, т. е. такой, что всякая другая задача из класса NP может быть сведена к ней за полиномиальное время.

Следует отметить, что в то же время Л. А. Левин в [2] независимо доказал теорему о полиномиальной сводимости, аналогичную теореме Кука.

Вскоре Р. М. Карп опубликовал работу [3], в которой показано, что многие известные комбинаторные задачи также являются NP-полными.

В 1979 г. вышла в свет богатая содержанием монография М. Гэри и Д. Джонсона [4] по теории полиномиальной сводимости дискретных задач. В ней содержится уникальное приложение, в котором собрано более трехсот NP-полных задач из различных областей науки. K настоящему времени список таких задач существенно расширен.

Из NP-полноты задачи о выполнимости следует, что если эта задача может быть решена за полиномиальное время, то и любая задача из класса NP полиномиально разрешима, и если какая-то задача из класса NP трудноразрешима, то любая NP-полная задача также должна быть трудноразрешимой.

Таким образом, если бы удалось доказать, что хотя бы какая-то одна NP-полная задача принадлежит классу $\mathrm{P}$, то тем самым было бы доказано, что $\mathrm{P}=\mathrm{NP}$, 
и можно было бы надеяться на возможность построения эффективных алгоритмов для решения многих дискретных задач. Однако до сих пор таких задач не обнаружено.

Проблема состоит в доказательстве того, что некоторая конкретная NP-полная задача не принадлежит классу Р.

\section{2. Изоморфизм неориентированных графов}

Пусть NP1 обозначает класс задач из NP, которые не являются ни полиномиальными, ни NP-полными (см. предыдущий раздел). Непустота класса NP1 (в предположении, что P $\neq$ NP) следует из работы [1]. Возникает вопрос: существуют ли "естественные" задачи, являющиеся кандидатами на принадлежность классу NP1?

Некоторое время таким кандидатом была задача состАВноЕ число, которая формулируется следующим образом [2].

УСловиЕ. Задано произвольное положительное число $N$.

Вопрос. Существуют ли такие целые числа $m$ u $n \quad(m, n>1)$, что $N=m n ?$

В 2004 г. в [3] доказано, что задача состАвноЕ число является полиномиальной, т. е. не принадлежит классу NP1.

До сих пор неизвестен статус задачи изомоРфизм грАФов, т. е. неизвестно, является ли полиномиальной, NP-полной или принадлежит классу NP1 следующая задача.

УСловИЕ. Заданы неориентированные графы $G_{1}=\left(V, E_{1}\right)$ u $G_{2}=\left(V, E_{2}\right)$.

Вопрос. Верно ли, что графы $G_{1}$ и $G_{2}$ изоморфны, т.е. существует ли взаимно однозначное отображение вершин графа $G_{1}$ на вершины графа $G_{2}$ такое, что две вершины в $G_{1}$ смежны тогда и только тогда, когда смежны их образы в $G_{2}$ ?

Проблема состоит в ответе на этот вопрос.

ЗАМЕчАНИЕ. Известно, что Задача ИЗОмОРФИЗм ГРАФОВ является полиномиальной для некоторых специальных классов графов, например, для плоских графов [4].

\section{3. Нижние оценки сложности схем, вычисляющих индивидуальные булевы функции}

Проблема нижних оценок сложности вычислений, имеющая более чем полувековую историю, - одна из наиболее трудных в дискретной математике. Она представляет большой интерес как для теории, так и для практики.

В прикладном плане решение проблемы нижних оценок сложности вычислений способствовало бы разработке более эффективных алгоритмов и созданию методики по оценке качества алгоритмов и программ.

Проблема нижних оценок сложности вычислений состоит, например, в том, чтобы установить хорошие нижние оценки сложности вычислений конкретных 
функций. Несмотря на значительные усилия многих математиков в течение нескольких десятилетий, нет заметного прогресса в решение этой проблемы.

Так обстоит дело в случае вычисления функций булевыми схемами, которые определяются следующим образом.

Пусть $\mathscr{E}=\left\{f_{1}, \ldots, f_{r}\right\}-$ произвольное множество булевых функций, называемое базисом. Булевой схемой (схемой из функииональных элементов) называется ориентированный граф без циклов с помеченными дугами и вершинами, множество вершин которого разбито на два подмножества. Вершины одного подмножества называются входами схемы. Им не инцидентны входящие дуги, и каждой такой вершине приписана буква алфавита переменных $X=\left\{x_{1}, \ldots, x_{n}\right\}$, каждой вершине второго подмножества приписан символ из базиса $\mathscr{E}$ (одна или несколько вершин объявляются выходами схемы). Число дуг, которые направлены в вершину, помеченную символом $f_{i}$, равно числу переменных функции $f_{i}$. Вершина с входящими в нее дугами называется функциональным (булевым) элементом. Каждый функциональный элемент реализует (вычисляет) функцию, которая ему приписана, а на выходе схемы вычисляется исходная булева функция.

Сложностью схемы называется число ее вершин, помеченных символами из $\mathscr{E}$.

В случае полных базисов $\mathscr{E}$ известны лишь линейные (относительно числа переменных вычисляемой булевой функции) нижние оценки сложности минимальных булевых схем.

Трудности решения проблемы нижних оценок сложности схем побудили рассматривать более слабые модели вычислений, а именно булевы схемы с ограничениями, схемы в неполных базисах и т.д. В результате исследований для таких схем удалось получить более высокие нижние оценки их сложности (см., например, работы [1]-[4]).

Высокие нижние оценки (более чем полиномиальные) получены А. А. Разборовым [5], [6] и А. Е. Андреевым [7], [8] для сложности минимальных булевых схем в базисе $\{\&, \vee\}$, которые вычисляют такие известные булевы функции, как перманент квадратных булевых матриц, функции, распознающие наличие клики заданного размера в неориентированных графах, и некоторые другие функции (в любом полном базисе эти функции вычисляются просто).

Задача состоит в указании конкретных булевых функций от $n$ переменных и доказательстве того, что сложность вычисления этих функций произвольными булевыми схемами не меньше $n^{3}, n^{4}$ и т. д.

\section{4. Сложность дизъюнктивных нормальных форм большинства булевых функций}

Литералом называется булева переменная или ее отрицание.

Элементарной конгюнкиией называется логическое произведение различных литералов, в котором отсутствуют литералы вида $x$ и $\bar{x}$.

Дизъюнктивной нормальной формой (д. н. ф.) называется дизъюнкция различных элементарных конъюнкций. Ясно, что каждая д.н. ф. задает (реализует) некоторую булеву функцию, т. е. функцию, определенную на множестве 
двоичных наборов и принимающую на каждом из этих наборов значение 0 или 1.

Д. н. ф. $D$, задающая булеву функцию $f$, называется кратчайшей, если в $D$ содержится наименьшее число конъюнкций среди всех д. н. ф., задающих $f$.

Пусть $f\left(x_{1}, \ldots, x_{n}\right)$ - произвольная булева функция от $n$ переменных; $m(f)$ сложность (число конъюнкций в кратчайшей д. н. ф.) функции $f$, и пусть

$$
m(n)=\max m\left(f\left(x_{1}, \ldots, x_{n}\right)\right),
$$

где максимум берется по всем булевым функциям от $n$ переменных. О. Б. Лупанов в [1] показал, что $m(n)=2^{n-1}$. Этот максимум достигается на следующих функциях:

$$
\begin{aligned}
& f_{1}\left(x_{1}, \ldots, x_{n}\right)=x_{1}+\cdots+x_{n} \equiv 0(\bmod 2), \\
& f_{2}\left(x_{1}, \ldots, x_{n}\right)=x_{1}+\cdots+x_{n}+1 \equiv 0(\bmod 2) .
\end{aligned}
$$

Вместе с тем В. В. Глаголев в [2] показал, что для почти каждой булевой функции $f\left(x_{1}, \ldots, x_{n}\right)$ справедливо неравенство

$$
m\left(f\left(x_{1}, \ldots, x_{n}\right)\right)>\frac{1-\varepsilon_{n}}{\log _{2} n \cdot \log _{2} \log _{2} n} \cdot 2^{n-1},
$$

где $\varepsilon_{n} \rightarrow 0$ при $n \rightarrow \infty$. Позднее С. Е. Кузнецов [3] улучшил эту оценку до следующей:

$$
m\left(f\left(x_{1}, \ldots, x_{n}\right)\right)>\frac{1-\varepsilon_{n}}{\log _{2} n \cdot \log _{2} \log _{2} n} \cdot 2^{n},
$$

где $\varepsilon_{n} \rightarrow 0$ при $n \rightarrow \infty$. Следовательно, для почти всех булевых функций от $n$ переменных сложности их кратчайших д. н. ф. заключены в интервале

$$
\left[\frac{1-o(1)}{\log _{2} n \cdot \log _{2} \log _{2} n} \cdot 2^{n}, 2^{n-1}\right] .
$$

Однако оказалось, что случаи, когда сложность $m\left(f\left(x_{1}, \ldots, x_{n}\right)\right)$ близка к $2^{n-1}$, являются нетипичными. Этот факт установил В. В. Глаголев в [4]: для почти каждой булевой функции $f\left(x_{1}, \ldots, x_{n}\right)$ справедливо неравенство

$$
m\left(f\left(x_{1}, \ldots, x_{n}\right)\right) \leqslant \frac{c \log _{2} \log _{2} n}{\log _{2} n} \cdot 2^{n} .
$$

В 1969 г. автор в [5] усилил этот результат до следующего: для почти каждой булевой функции $f\left(x_{1}, \ldots, x_{n}\right)$ справедливо неравенство

$$
m\left(f\left(x_{1}, \ldots, x_{n}\right)\right)<\frac{2^{n+2}}{\log _{2} n} .
$$

Аналогичный результат другим методом получил А. А. Сапоженко [6].

Наконец, в [7], [8] автором был предложен метод построения д. н. ф., из которого извлекается следующий результат: для почти любой булевой функции $f\left(x_{1}, \ldots, x_{n}\right)$ сложность кратчайшей д. н. ф. $m\left(f\left(x_{1}, \ldots, x_{n}\right)\right)$ удовлетворяет неравенству

$$
m\left(f\left(x_{1}, \ldots, x_{n}\right)\right)<2.63 \cdot \frac{2^{n}}{\log _{2} n \cdot \log _{2} \log _{2} n} .
$$


ЗАмЕчАнИЕ. После выхода в свет статьи [7] появилась публикация А. Е. Андреева [9], в которой приведен (без полного доказательства) результат, аналогичный результату из [7].

Из (1) и (2) следует, что сложность кратчайших д. н. ф. почти каждой булевой функции от $n$ переменных по порядку равна $2^{n} /\left(\log _{2} n \cdot \log _{2} \log _{2} n\right)$. Вместе с тем Р.Г. Нигматуллин [10] с использованием введенного им вариационного принципа доказал, что для почти всех булевых функций $f\left(x_{1}, \ldots, x_{n}\right)$ величины $m\left(f\left(x_{1}, \ldots, x_{n}\right)\right)$ асимптотически совпадают.

Проблема состоит в нахождении явного вида этой асимптотики при $n \rightarrow \infty$.

\section{5. Сложность покрытий числовых множеств арифметическими прогрессиями}

Пусть $M_{n}=\{1, \ldots, n\}, R$ - произвольное подмножество из $M_{n}$ и $A(R)$ - минимальное число арифметических прогрессий, теоретико-множественная сумма которых совпадает с $R$. Величина $A(R)$ называется сложностъю покрытия множества $R$ арифметическими прогрессиями.

Первые результаты по определению величины $A(R)$ получили С. С. Кислицын и А. А. Сапоженко. В [1] они установили следующие факты:

а) для почти каждого подмножества $R$ из $M_{n}$ справедливо неравенство

$$
A(R) \geqslant \frac{n}{3 \log _{2} n}
$$

б) для почти каждого подмножества $R$ из $M_{n}$ справедливо неравенство

$$
a(R)<\frac{n \ln 2 \cdot \log _{2} \log _{2} n}{2 \log _{2} n}(1+o(1)) .
$$

Позднее оценка (1) была улучшена в работе [2] до следующей: для почти каждого подмножества $R$ из $M_{n}$ справедливо неравенство

$$
A(R) \geqslant \frac{n}{2 \log _{2} n-1} .
$$

ЗАмЕчАнИЕ. В. В. Глаголев [3] рассмотрел эту задачу со следующим дополнительным ограничением: все прогрессии, образующие покрытия множества $R$, должны иметь одну и ту же разность. Им было показано, что в наилучшем покрытии "самого плохого подмножества из $M_{n}$ " число арифметических прогрессий асимптотически равно $n / 4$.

А. Д. Коршунов в [4] усилил результаты из [1], [2] до следующих:

а) для почти каждого подмножества $R$ из $M_{n}$ справедливо неравенство

$$
A(R) \geqslant \frac{n}{\log _{2} n}(1-\varepsilon(n)),
$$

где $\varepsilon(n) \rightarrow \infty$ при $n \rightarrow \infty$;

б) для почти каждого подмножества $R$ из $M_{n}$ справедливо неравенство

$$
a(R)<\frac{1.6 n}{\log _{2} n}(1+o(1)) .
$$


Из утверждений (2) и (3) следует, что сложность покрытия почти каждого подмножества $R$ из $M_{n}$ арифметическими прогрессиями по порядку равна $n / \log _{2} n$.

Проблема состоит в нахождении асимптотического поведения величины $A(R)$ для почти всех подмножеств $R$ из $M_{n}$ при $n \rightarrow \infty$.

\section{6. Протыкание подкубов $n$-мерного булева куба}

Пусть $E^{n}$ обозначает $n$-мерный булев (единичный) куб, т. е. неориентированный граф с $2^{n}$ вершинами, которые помечены двоичными наборами длины $n$. В этом графе вершины $\widetilde{\alpha}=\left(\alpha_{1}, \ldots, \alpha_{n}\right)$ и $\widetilde{\beta}=\left(\beta_{1}, \ldots, \beta_{n}\right)$ смежны (соединены ребром) тогда и только тогда, когда наборы $\widetilde{\alpha}$ и $\widetilde{\beta}$ различаются точно в одной координате.

Множество вершин $\left\{\widetilde{\alpha}_{1}, \ldots, \widetilde{\alpha}_{2^{k}}\right\}$ из $E^{n}$, которые не отличаются друг от друга в $n-k$ произвольно выбранных координатах, а значения остальных координат произвольны, называется $k$-мерным подкубом (интервалом ранга $n-k$ ) в $E^{n}$.

В начале 1960-х годов О.Б. Лупанов сформулировал следующую задачу о протыкании $k$-мерных подкубов в $E^{n}$. При каждом фиксированном $k, 0 \leqslant$ $k \leqslant n$, найти в $E^{n}$ минимальное по размеру множество вершин $B(n, k)$, протыкающее все $k$-мерные подкубы в $E^{n}$.

Легко видеть, что при любом $n=1,2, \ldots$

$$
|B(n, 0)|=2^{n}, \quad|B(n, 1)|=2^{n-1}, \quad|B(n, n-1)|=2, \quad|B(n, n)|=1
$$

и

$$
|B(n, k)| \geqslant 2^{n-k} \quad \text { при произвольном } k, 0 \leqslant k \leqslant n .
$$

О. Б. Лупанов показал (не опубликовано), что при $n \rightarrow \infty$

$$
|B(n, n-2)| \sim \log _{2} n .
$$

Случай $k=2$ рассмотрела Е. А. Косточка. Она в [1] показала, что

$$
|B(n, 2)|=\left\lfloor\frac{2^{n}}{3}\right\rfloor .
$$

В случае произвольного $k, 3 \leqslant k \leqslant n-3$, Э. И. Нечипорук в [2] (см. также [3]) доказал, что

$$
|B(n, k)|<2^{n-k}\left(\ln \left(\begin{array}{l}
n \\
k
\end{array}\right)+1\right)+1 .
$$

Наконец, В. К. Леонтьев [4] показал, что в случае больших $n$ и при произвольном $k, 3 \leqslant k \leqslant n-3$, для размера $B(n, k)$ справедливо неравенство

$$
|B(n, k)| \geqslant 2^{n-k-2} \log _{2} n .
$$

Поскольку верхняя и нижняя оценки (1), (2) для $|B(n, k)|$ при многих $k$ существенно отличаются друг от друга (например, при $k=\lfloor n / 2\rfloor$ и $n \rightarrow \infty)$, то остается нерешенной задача о нахождении точного (или асимптотического) значения величины $|B(n, k)|$ при $n \rightarrow \infty$ и произвольном $k, 3 \leqslant k \leqslant n-3$. 


\section{7. Длина максимальной змеи в $n$-мерном булевом кубе}

Пусть $\rho(\widetilde{\alpha}, \widetilde{\beta})=\sum_{i=1}^{n}\left|\alpha_{i}-\beta_{i}\right|$ есть расстояние Хемминга между вершинами $\widetilde{\alpha}=\left(\alpha_{1}, \ldots, \alpha_{n}\right)$ и $\widetilde{\beta}=\left(\beta_{1}, \ldots, \beta_{n}\right)$ из $E^{n}$. Конечная последовательность $\widetilde{\alpha}_{1}, \ldots, \widetilde{\alpha}_{m}$ вершин в $n$-мерном булевом кубе $E^{n}$ называется змеей, если

1) $\rho\left(\widetilde{\alpha}_{i}, \widetilde{\alpha}_{i+1}\right)=1$ при каждом $i=1, \ldots, m-1$ и $\rho\left(\widetilde{\alpha}_{1}, \widetilde{\alpha}_{m}\right)=1$,

2) $\rho\left(\widetilde{\alpha}_{i}, \widetilde{\alpha}_{j}\right)>1$ при любых $i$ и $j$ таких, что $|i-j|>1$ и $|m-j| \neq|m-1|$.

Иначе говоря, змея в $E^{n}$ является простым циклом без хорд. Число вершин змеи называется ее длиной.

Пусть $l(n)$ обозначает длину максимальной змеи в $E^{n}$. Задача состоит в нахождении величины $l(n)$.

В настоящее время величина $l(n)$ известна только при $n=2,3,4,5,6$ и 7. В общем случае величина $l(n)$ неизвестна.

Впервые задача об определении длины максимальной змеи в $E^{n}$ была сформулирована в работе [1] в связи с построением кодов, исправляющих ошибки, для специальных аналого-цифровых систем. В дальнейшем эта задача рассматривалась в связи с выяснением трудностей минимизации дизъюнктивных нормальных форм булевых функций локальными алгоритмами [2].

Определением величины $l(n)$ занимались многие исследователи. K настоящему времени опубликовано более 50 работ.

Сначала коснемся нижних оценок для $l(n)$. В [1] показано, что $l(n)>c \cdot 2^{n / 2}$, где $c$ - абсолютная положительная константа. Дальнейшее продвижение в существенном повышении нижней оценки для $l(n)$ содержится в работе Ю. Л. Васильева [3]. Им показано, что

$$
l(n)> \begin{cases}\frac{2^{n}}{n}, & \text { если } n \text { является степенью } 2, \\ (1-\varepsilon(n)) \frac{2^{n-1}}{n} & \text { в противном случае }\end{cases}
$$

где $\varepsilon(n) \rightarrow 0$ при $n \rightarrow \infty$.

А. А. Евдокимов в [4] доказал, что

$$
l(n) \geqslant 2^{n-11}-4 .
$$

Более точная оценка вида

$$
l(n) \geqslant 4.2\left(2^{n-1}-1\right)
$$

была установлена им в [5].

В настоящее время наилучшей нижней оценкой для $l(n)$ является оценка

$$
l(n) \geqslant \frac{27}{256} \cdot 2^{n}
$$

опубликованная в [6].

Другие нижние оценки, не превосходящие (1), опубликовали: H. L. Abbott (1965), R. C. Singleton (1966), L. Danzer \& V. Klee (1967), J. Wojciechowski (1989), R. Röpling-Lenhart (1991) и другие. 
Теперь перейдем к освещению верхних оценок для $l(n)$. Легко видеть, что

$$
l(n) \leqslant \frac{n}{n-1} \cdot 2^{n-1} .
$$

В самом деле, пусть произвольная змея в $E^{n}$ состоит из наборов множества $M=\left\{\widetilde{\alpha}_{1}, \ldots, \widetilde{\alpha}_{m}\right\}$. Тогда число ребер в $E^{n}$, которые соединяют вершины из $M$ с вершинами из $E^{n} \backslash M$, равно $m(n-2)$. Вместе с тем поскольку степень каждой вершины из $E^{n}$ равна $n$, а некоторые ребра соединяют вершины из $E^{n} \backslash M$, имеем

$$
m(n-2) \leqslant n\left|E^{n} \backslash M\right|=n\left(2^{n}-m\right) .
$$

Следовательно,

$$
m \leqslant \frac{n}{n-1} 2^{n-1},
$$

т. е. справедливо (2). Оценка (2) содержится в [1].

Лучшие верхние оценки для $l(n)$ получали многие исследователи. Первую оценку, меньшую $2^{n-1}$, получил В.В. Глаголев. В [7] им показано, что при любом $n \geqslant 5$

$$
l(n)<2^{n-1} .
$$

Наилучшая известная оценка содержится в работе [8]:

$$
l(n) \leqslant 2^{n-1}\left(1-\frac{1}{89 \sqrt{n}}+O\left(\frac{1}{n}\right)\right) .
$$

Другие верхние оценки для $l(n)$, не меньшие оценки (3), опубликовали: L. Danzer \& V. Klee (1967), R. J. Douglas (1969), А. А. Евдокимов (1971), K. Deimer (1985), H. S. Snevily (1994), П. Г. Емельянов (1995), P. G. Emelyanov \& A. Lukito (2000) и другие.

Поскольку нижняя и верхняя оценки $(1),(3)$ для $l(n)$ совпадают только с точностью до порядка, остается нерешенной задача о нахождении асимптотического поведения величины $l(n)$ при $n \rightarrow \infty$.

\section{8. Гамильтоновы циклы в $n$-мерном булевом кубе}

Пусть $E^{n}-n$-мерный булев куб. Замкнутый маршрут без самопересечений в $E^{n}$ называется гамильтоновым циклом, если в нем содержатся все вершины из $E^{n}$.

Пусть $h(n)$ обозначает число гамильтоновых циклов в $E^{n}$. Задача определения числа $h(n)$ при любом $n \geqslant 2$ поставлена в [1]. Она изучалась многими исследователями (см., например, работы [2]-[4]).

Тривиальная верхняя оценка

$$
h(n)<n^{2^{n}}
$$

следует из того, что если в $E^{n}$ зафиксировать произвольную вершину (например, вершину $\widetilde{0}=(0, \ldots, 0))$ и гамильтоновы циклы получать методом последовательного удлинения простых цепей (начинающихся в вершине $\widetilde{0}$ ), то на каждом шаге имеющуюся цепь можно удлинять не более чем $n$ способами. 
Более точную верхнюю оценку для $h(n)$ получили А.Л. Пережогин и В. Н. Потапов. В работе [5] они показали, что при $n \rightarrow \infty$

$$
\ln h(n) \leqslant 2^{n}(\ln n-1+o(1)) .
$$

Наилучшая нижняя оценка для $h(n)$ содержится в работе [6]. В ней показано, что

$$
\ln h(n) \geqslant 2^{n}(\ln n+\ln \ln 2-10 \ln \ln \ln n-o(1)) .
$$

Из верхней и нижней оценок (1) и (2) для $\ln h(n)$ следует, что при $n \rightarrow \infty$

$$
\ln h(n) \sim 2^{n} \ln n .
$$

Остаются нерешенными следующие более сложные задачи:

а) определить порядок функции $h(n)$;

б) найти асимптотику функции $h(n)$.

\section{9. Проблема гамильтоновости двух средних слоев $n$-мерного куба}

Весом вершины $\widetilde{\alpha}=\left(\alpha_{1}, \ldots, \alpha_{n}\right)$ в булевом кубе $E^{n}$ называется число единиц в $\widetilde{\alpha}$. При нечетном $n \geqslant 3$ обозначим через $G_{n}$ такой подграф в $E^{n}$, в котором имеется $\left(\begin{array}{c}n \\ (n-1) / 2\end{array}\right)$ вершин веса $(n-1) / 2$ и $\left(\begin{array}{c}n \\ (n+1) / 2\end{array}\right)$ вершин веса $(n+1) / 2$. Иначе говоря, вершинами графа $G_{n}$ являются все вершины двух средних слоев булева куба $E^{n}$ нечетной размерности.

Гипотеза состоит в том, что при любом нечетном $n \geqslant 3$ граф $G_{n}$ является гамильтоновым.

Исследованием этой гипотезы занимались многие (см., например, работы [1]-[3]). Справедлива ли гипотеза о существовании гамильтонова цикла в графе $G_{n}$ при любом нечетном $n \geqslant 3$, до сих пор неизвестно.

\section{0. Совершенные двоичные $(n, 3)$-коды}

Пусть $\rho(\widetilde{\alpha}, \widetilde{\beta})=\sum_{\widetilde{\beta}=1}^{n}\left|\alpha_{i}-\beta_{i}\right|$ есть расстояние Хемминга между вершинами $\widetilde{\alpha}=\left(\alpha_{1}, \ldots, \alpha_{n}\right)$ и $\widetilde{\beta}=\left(\beta_{1}, \ldots, \beta_{n}\right)$ из $E^{n}$.

Множество $C$ вершин из $E^{n}$ называется совершенным (плотно упакованным) двоичным $(n, 3)$-кодом, если шары радиуса 1 с центрами из $C$ не пересекаются, а множество вершин, принадлежащих их объединению, совпадает с множеством вершин из $E^{n}$.

Так как каждому шару радиуса 1 принадлежит $n+1$ вершина из $E^{n}$, то число $2^{n} /(n+1)$ должно быть целым. Поэтому совершенный двоичный $(n, 3)$-код может существовать только тогда, когда число $n+1$ равно степени 2 . Оказывается, что при любом таком $n$ совершенный $(n, 3)$-код существует. Впервые такие коды описал в 1950 г. Р. Хемминг [1] (они называются кодами Хемминга).

Пусть $B(n)$ обозначает множество всех совершенных двоичных $(n, 3)$-кодов длины $n$. В 1962 г. Ю. Л. Васильев в [2] предложил метод построения большого числа таких кодов и доказал, что при $n+1=2^{k}, k=2,3, \ldots$, и $n \rightarrow \infty$

$$
|B(n)|>2^{2^{\frac{n+1}{2}(1-o(1))}} .
$$


В настоящее время известно несколько различных методов построения совершенных двоичных $(n, 3)$-кодов (см., например, работы [3]-[5]). Эти методы позволяют получать такое количество кодов, которое незначительно отличается от нижней оценки Васильева и имеет тот же вид (1). Так как число совершенных двоичных $(n, 3)$-кодов меньше числа всех $2^{n} /(n+1)$-вершинных подмножеств в $E^{n}$, то

$$
|B(n)|<\left(\begin{array}{c}
2^{n} \\
2^{n} /(n+1)
\end{array}\right)=2^{2^{n(1-o(1))}} .
$$

Более точную верхнюю оценку для $|B(n)|$ получил С. В. Августинович [6]. Однако она имеет тот же вид, что и правая часть из $(2)$.

Нижняя и верхняя оценки для $|B(n)|$ из (1) и (2) существенно различаются. Поэтому из них не извлекается информация об истинном значении величины $|B(n)|$. В связи с этим представляет интерес задача о выяснении асимптотического поведения величины $\log _{2} \log _{2}|B(n)|$. Более сложной является задача нахождения асимптотики величины $\log _{2}|B(n)|$. Еще более сложной является задача нахождения асимптотики размера множества $B(n)$.

\section{1. Пороговые булевы функции}

Булева функция $f\left(x_{1}, \ldots, x_{n}\right)$ называется пороговой, если существуют вещественные числа $a_{1}, \ldots, a_{n}, b$ такие, что двоичный набор $\left(\alpha_{1}, \ldots, \alpha_{n}\right)$ является решением неравенства $a_{1} x_{1}+\cdots+a_{n} x_{n} \geqslant b$ тогда и только тогда, когда $f\left(\alpha_{1}, \ldots, \alpha_{n}\right)=1$. Коэффициенты $a_{i}$ называются весами, а число $b-$ порогом.

Небольшим изменением порога и весов можно добиться строгого неравенства. Таким образом, пороговая булева функция задается гиперплоскостью, рассекающей булев куб так, что в вершинах по одну сторону гиперплоскости функция равна нулю, по другую - единице.

Такой взгляд оказывается весьма полезным в теоретических исследованиях, так как позволяет использовать геометрическую интуицию при решении различных задач, связанных с пороговыми функциями. Подробные сведения о пороговых функциях изложены в обзорной статье Ю. А. Зуева [1].

Пусть $T(n)$ обозначает число пороговых булевых функций от $n$ переменных. К вычислению этой функции приступили в конце пятидесятых годов прошлого века. К 1965 г. удалось получить следующие результаты.

Верхняя оценка вида

$$
T(n)<2^{n^{2}(1-o(1))}
$$

получена в [2] (см. также [3]).

Нижняя оценка для числа пороговых булевых функций от $n$ переменных вида

$$
T(n)>2^{\frac{1}{2} n^{2}(1-o(1))}
$$

впервые опубликована в работе [4]. Позднее Ю. А. Зуев в работе [5] (см. также [6]) улучшил оценку (2) до следующей:

$$
T(n)>2^{n^{2}(1-10 / \ln n)} .
$$

В 1992 г. оценка (3) была улучшена А. А. Ирматовым в работе [7]. 
Из (1) и (3) следует асимптотика для логарифма числа пороговых булевых функций от $n$ переменных при $n \rightarrow \infty$

$$
\log _{2} T(n) \sim n^{2}
$$

Теперь очередная задача состоит в нахождении асимптотики функции $T(n)$ при $n \rightarrow \infty$.

\section{2. Число систем троек Штейнера}

Cистемой троек Штейнера $S$ на $n$-элементном множестве $M$ называется такое семейство $\mathscr{F}$ трехэлементных подмножеств из $M$, что каждая пара элементов из $S$ принадлежит точно одному подмножеству из $\mathscr{F}$. Размер множества $M$ называется порядком системы. Известно (см., например, [1]), что система троек Штейнера порядка $n$ существует тогда и только тогда, когда

$$
n \equiv 1 \text { или } 3(\bmod 6) .
$$

Такие $n$ назовем значащими.

Пусть $S(n)$ обозначает число неизоморфных (т. е. неэквивалентных относительно действующей на $M$ симметрической группы) систем троек Штейнера порядка $n$. Проблема определения $S(n)$ была поставлена Дж. Штейнером в 1853 г. Имеется большое число публикаций, в которых содержатся точные значения функции $S(n)$ при различных конкретных $n$.

Нахождением верхних и нижних оценок для $S(n)$ занималось несколько исследователей (В. Е. Алексеев, E. Assmus \& H. F. Mattson, J. Doyen, G. Valette и другие).

Наилучшая известная верхняя оценка имеет вид:

$$
S(n)<n^{n^{2} / 6-O(n)} .
$$

Она получена в [2].

Для значащих $n$ наилучшая известная нижняя оценка

$$
S(n)>\left(e^{-5} n\right)^{n^{2} / 6}
$$

получена Г. П. Егорычевым в [3].

Из неравенств (1) и (2) следует, что при $n \rightarrow \infty$ асимптотика логарифма функции $S(n)$ равна $n^{2} \log _{2} n$.

Остается нерешенной задача о нахождении асимптотики функции $S(n)$ при $n \rightarrow \infty$.

\section{3. Восстановление графа по подграфам}

Проблема изоморфизма для неориентированных графов $G_{1}$ и $G_{2}$ с одинаковым числом вершин состоит в определении того, существует ли взаимно однозначное отображение вершин графа $G_{1}$ на вершины графа $G_{2}$ такое, что две вершины в $G_{1}$ смежны тогда и только тогда, когда смежны их образы в $G_{2}$. 
Граф $H$ называется порожденным подграфом неориентированного графа $G$, если множество вершин графа $H$ является подмножеством множества вершин графа $G$, а множество ребер графа $H$ совпадает с множеством тех ребер графа $G$, оба конца которых принадлежат графу $H$.

Пусть $G$ - произвольный $n$-вершинный неориентированный граф и $G_{1}, \ldots, G_{n}$ - множество всех $(n-1)$-вершинных подграфов графа $G$ таких, что граф $G_{i}$ получается путем удаления из $G i$-й вершины и инцидентных ей ребер.

С. Улам [1] поставил следующий вопрос: верно ли, что $n$-вершинный граф $G$ восстанавливается (с точностью до изоморфизма) по $(n-1)$-вершинным подграфам $G_{1}, \ldots, G_{n}$, в которых вершины не помечены? Для некоторых классов графов ответ положителен. Так, например, в [2] дан положительный ответ на этот вопрос для деревьев. Однако ответ на этот вопрос в общем случае до сих пор неизвестен. Каков этот ответ? Проблема Клама изучается также в [3]-[7].

Выражаю искреннюю благодарность С.И. Адяну за проявленный интерес к настоящей работе и ценные замечания.

\section{Список литературы}

\section{К разделу 1}

[1] S. A. Cook, "The complexity of theorem-proving procedures", Proceedings of the 3rd Annual ACM Symposium on the Theory of Computing (Shaker Heights, 1971), ACM Press, New York, 1971, 151-158; pус. пер.: С. А. Кук, "Сложность процедур вывода теорем", Кибернет. сб. Нов. сер., 1975, № 12, 5-15.

[2] Л. А. Левин, "Универсальные задачи перебора", Проблемы передачи информации, 9:3 (1973), 115-116; англ. пер.: L. A. Levin, "Universal problems of full search", Probl. Inf. Transm., 9:3 (1973), 265-266.

[3] R. M. Karp, "Reducibility among combinatorial problems", Complexity of computer computations, Proc. Sympos. (IBM Thomas J. Watson Res. Center, Yorktown Heights, N.Y., 1972), Plenum, New York, 1972, 85-103; рус. пер.: Р. М. Карп, "Сводимость комбинаторных задач", Кибернет. сб. Нов. сер., 1975, № 12, 16-38.

[4] M. R. Garey, D.S. Johnson, Computers and intractability. A guide to the theory of NP-completeness, A Series of Books in the Mathematical Sciences, Freeman, San Franсіsсо, 1979; рус. пер.: М. Гэри, Д. Джонсон, Вычислителъные машины и труднорешаемые задачи, Мир, М., 1982.

\section{К разделу 2}

[1] R. E. Ladner, "On the structure of polynomial time reducibility", J. Assoc. Comput. Mach., 22:1 (1975), 155-171.

[2] M.R. Garey, D. S. Johnson, Computers and intractability. A guide to the theory of NP-completeness, A Series of Books in the Mathematical Sciences, Freeman, San Franсіsсо, 1979; рус. пер.: М. Гэри, Д. Джонсон, Вычислительные машины и труднорешаемые задачи, Мир, М., 1982.

[3] M. Agrawal, N. Kayal, N. Saxena, "PRIMES is in P", Ann. of Math., 160:2 (2004), 781-793.

[4] J.E. Hopcroft, R.E. Tarjan, "Isomorphism of planar graphs", Complexity of computer computations, Proc. Sympos. (IBM Thomas J. Watson Res. Center, Yorktown Heights, N.Y., 1972), Plenum, New York, 1972, 131-152, 187-212; рус. пер.: 
Дж. Е. Хокрофт, Р.Е. Тарьян, "Изоморфизм планарных графов", Кибернет. сб. Нов. сер., 1975, № 12, 39-61.

\section{К разделу 3}

[1] Б.А. Субботовская, "О реализации линейных функций формулами в базисе $\left\{\&, \vee,^{-}\right\} "$, Докл. АН СCCP, $136: 3$ (1961), 553-555; англ. пер.: В. A. Subbotovskaya, "Realization of linear functions by formulas using $\{\&, \vee,-\}$ ", Sov. Math., Dokl., 2 (1961), 110-112.

[2] Б. А. Субботовская, "О сравнении базисов при реализации функций алгебры логики формулами", Докл. АН CCCP, 4 (1963), 784-787; англ. пер.: В. A. Subbotovskaya, "Comparison of bases in the realization by formulas of functions of the algebra of logic", Sov. Math., Dokl., 149:4 (1963), 478-481.

[3] Э. И. Нечипорук, “Об одной булевой функции”, Докл. АН СССР, 169:4 (1966), 765-766; англ. пер.: Eh. I. Nechiporuk, "A Boolean function", Sov. Math., Dokl., 7 (1966), 999-1000.

[4] А. Е. Андреев, "Об одном методе получения более чем квадратичных эффективных нижних оценок сложности $\pi$-схем", Вестник МГУ. Сер. 1. Матем., мех., 1987, № 1, 70-73; англ. пер.: А. E. Andreev, "A method for obtaining efficient lower estimates of complexity of $\pi$-schemes", Mosc. Univ. Math. Bull., 42:1 (1987), 63-66.

[5] А.А. Разборов, "Нижние оценки монотонной сложности некоторых булевых функций”, Докл. АН СCCP, 281:4 (1985), 798-801; англ. пер.: А. A. Razborov, "Lower bounds for the monotone complexity of some Boolean functions", Sov. Math., Dokl., 31 (1985), 354-357.

[6] А. А. Разборов, "Нижние оценки монотонной сложности логического перманента", Матем. заметки, 37:6 (1985), 887-900; англ. пер.: А. A. Razborov, "Lower bounds on monotone complexity of the logical permanent", Math. Notes, 37:6 (1985), 485-493.

[7] А. Е. Андреев, "Об одном методе получения нижних оценок сложности индивидуальных монотонных функций”, Докл. АН СССР, 282:5 (1985), 1033-1037; англ. пер.: A. E. Andreev, "On a method for obtaining lower bounds for the complexity of individual monotone functions", Sov. Math., Dokl., 31 (1985), 530-534.

[8] А. Е. Андреев, "Об одном методе получения эффективных нижних оценок монотонной сложности", Алгебра и логика, 26:1 (1987), 3-26; англ. пер.: А. Е. Andreev, "A method for obtaining efficient lower bounds for monotone complexity", Algebra Logic, 26:1 (1987), 1-18.

\section{К разделу 4}

[1] О.Б. Лупанов, "О реализации функций алгебры логики формулами из конечных классов (формулами ограниченной глубины) в базисе $\{\&, \vee,-\} "$, Проблемь кибернетики, 1961, №6, 5-14; англ. пер.: О. B. Lupanov, "On the realization of functions of logical algebra by formulae of finite classes (formulae of limited depth) in the basis $\{\&, \vee,-\} "$, Probl. Cybernetics, 1965, № 6, 1-14.

[2] В. В. Глаголев, "Оценка сложности сокращенной дизъюнктивной нормальной формы для почти всех функций алгебры логики”, Докл. АН СCCP, 158:4 (1964), 770-773; англ. пер.: V. V. Glagolev, "Estimate of the complexity of a reduced disjunctive normal form for almost all functions of the algebra of logic", Sov. Math., Dokl., 5 (1965), 1302-1305.

[3] С.Е. Кузнецов, "О нижней оценке длины кратчайшей д.н.ф. почти всех булевых функций”, Вероятностные методы и кибернетика, №19, Изд-во Казанского ун-та, Казань, 1983, 44-47.

[4] В. В. Глаголев, "Верхняя оценка сложности минимальной д.н.ф. для почти всех функций алгебры логики", Дискретный анализ: Сб. науч. тр., № 5, Ин-т математики СО АН СССР, Новосибирск, 1965, 3-8. 
[5] А. Д. Коршунов, "Верхняя оценка сложности кратчайших д.н.ф. почти всех булевых функций", Кибернетика, 1969, № 6, 1-11; англ. пер.: A. D. Korshunov, "An upper bound of the complexity of the shortest disjunctive normal forms for almost all Boolean functions", Cybernetics, 5 (1969), 705-715.

[6] А. А. Сапоженко, "О сложности дизъюнктивных нормальных форм, полученных с помощью градиентного алгоритма", Дискретный анализ: Сб. науч. тр., № 21, Ин-т математики СО АН СССР, Новосибирск, 1972, 62-71.

[7] А. Д. Коршунов, "О сложности кратчайших дизъюнктивных нормальных форм булевых функций”, Методы дискретного анализа в изучении булевых функиий: Сб. науч. тр., № 37, Ин-т математики СО АН СССР, Новосибирск, 1981, 9-41; англ. пер.: А. D. Korshunov, "On the complexity of the shortest disjunctive normal forms of Boolean functions", Amer. Math. Soc. Transl., 135 (1987), 55-79.

[8] А. Д. Коршунов, "О сложности кратчайших дизъюнктивных нормальных форм случайных булевых функций”, Методы дискретного анализа в оптимизаиии управляюоих систем: Сб. науч. тр., № 40, Ин-т математики СО АН СССР, Новосибирск, 1983, 25-53.

[9] А.Е. Андреев, "О синтезе дизъюнктивных нормальных форм, близких к минимальным", Докл. АН СCCP, 269:1 (1983), 11-15; англ. пер.: A. E. Andreev, "On the synthesis of disjunctive normal forms which are close to minimal", Sov. Math., Dokl., 27:2 (1983), 265-269.

[10] Р. Г. Нигматуллин, "Вариационный принцип в алгебре логики”, Дискретный анализ: Сб. науч. тр., №10, Ин-т математики СО АН СССР, Новосибирск, 1967, 69-89.

\section{К разделу 5}

[1] С. С. Кислицын, А. А. Сапоженко, “О представлении множества чисел теоретикомножественной суммой арифметических прогрессий", Вопросы кибернетики, № 16, Научный совет АН СССР по комплексной проблеме "Кибернетика", М., $1975,53-62$.

[2] С. В. Новиков, С. В. Олексин, "О покрытии множества арифметическими прогрессиями", Изв. АН БССР. Сер. физ.-матем. наук, 1979, № 6, 25-27.

[3] В. В. Глаголев, "О покрытии арифметическими прогрессиями", Методы дискретного анализа в синтезе управляющих систем: Сб. науч. тр., № 32, Ин-т математики СО АН СССР, Новосибирск, 1978, 34-39.

[4] А. Д. Коршунов, "О сложности покрытий числовых множеств арифметическими прогрессиями”, Сиб. журн. исслед. опер., 1:2 (1994), 40-60; англ. пер.: A. D. Korshunov, "Complexity of coverings of number sets by arithmetical progressions", Discrete analysis and operations research, Math. Appl., 355, Kluwer, Dordrecht, 1996, 81-100.

\section{К разделу 6}

[1] Е. А. Косточка, "О прокалывании граней единичного $n$-мерного куба”, Дискретный анализ: Сб. науч. тр., №28, Ин-т математики СО АН СССР, Новосибирск, 1976, 55-64.

[2] Э. И. Нечипорук, "О сложности вентильных схем, реализующих булевские матрицы с неопределенными элементами”, Докл. АН СCCP, 163:1 (1965), 40-42; англ. пер.: E. I. Nechiporuk, "Complexity of gating circuits which are realized by Boolean matrices with undetermined elements", Sov. Phys., Dokl., 10 (1966), 591-593.

[3] Э. И. Нечипорук, "О топологических принципах самокорректирования", Проблемы кибернетики, № 21, Наука, М., 1969, 5-102.

[4] В. К. Леонтьев, "Верхняя оценка $\alpha$-глубины $(0,1)$-матриц", Матем. заметки, 15:3 (1974), 421-429; англ. пер.: V. K. Leont'ev, "An upper bound for the $\alpha$-height of (0,1)-matrices", Math. Notes, 15:3 (1974), 245-250. 


\section{К разделу 7}

[1] W. H. Kautz, "Unit distance error checking codes", IRE Trans. Electron. Comput., 7 (1958), 177-180.

[2] Ю. И. Журавлев, “Теоретико-множественные методы в алгебре логики”, Проблемы кибернетики, №8, Физматлит, М., 1962, 5-44; нем. пер.: Yu. I. Zhuravlev, "Mengentheoretische Methoden in der Aussagenlogik", Probl. Kybernetik, 8 (1965), $3-51$.

[3] Ю.Л. Васильев, "О длине цикла в $n$-мерном единичном кубе", Докл. АН CCCP, 148:4 (1963), 753-756; англ. пер.: Yu. L. Vasil'ev, "On the length of a cycle in an n-dimensional unit cube", Sov. Math., Dokl., 4 (1963), 160-163.

[4] А.А. Евдокимов, "О максимальной длине цепи в единичном $n$-мерном кубе", Матем. заметки, 6:3 (1969), 309-319; англ. пер.: A. A. Evdokimov, "Maximal length of circuit in a unitary n-dimensional cube", Math. Notes, 6:3 (1970), 642-648.

[5] А. А. Евдокимов, О максимальной длине цепи в п-мерном единичном кубе и некоmорых родственных задачах, Дис. ... канд. физ.-матем. наук, Ин-т математики СО АН СССР, Новосибирск, 1971.

[6] H. L. Abbott, M. Katchalski, "On the construction of snake in the box codes", Utilitas Math., 40 (1991), 97-116.

[7] В. В. Глаголев, "Верхняя оценка длины цикла в $n$-мерном единичном кубе”, Дискретныц анализ: Сб. науч. тр., №6, Ин-т математики СО АН СССР, Новосибирск, 1966, 3-7.

[8] G. Zémor, "An upper bound on the size of the snake-in-the-box", Combinatorica, 17:2 (1997), 287-298.

\section{К разделу 8}

[1] E. N. Gilbert, "Gray codes and paths on the n-cube", Bell System Tech. J., 37:3 (1958), 815-826.

[2] H. L. Abbott, "Hamiltonian circuits and paths on the n-cube", Canad. Math. Bull., 9:5 (1966), 557-562.

[3] E. Dixon, S. Goodman, "On the number of Hamiltonian circuits in the $n$-cube", Proc. Amer. Math. Soc., 50 (1975), 500-504.

[4] R. J. Douglas, "A note on a theorem of H. L. Abbott", Canad. Math. Bull., 13:1 (1970), 79-81.

[5] А. Л. Пережогин, В. Н. Потапов, “О числе гамильтоновых циклов в булевом кубе”, Дискрет. анализ и исслед. опер. Сер. 1, 8:2 (2001), 52-62.

[6] T. Feder, C. Subi, "Nearly tight bounds on the number of Hamiltonian circuits of the hypercube and generalizations", Inform. Process. Lett., 109:5 (2009), 267-272.

\section{К разделу 9}

[1] А. А. Евдокимов, А. Л. Пережогин, "Минимальные нумерации подмножеств конечного множества и проблема гамильтоновости графа средних слоев гиперкуба", Дискрет. анализ и исслед. опер. Сер. 1, 4:4 (1997), 6-12; англ. пер.: A. A. Evdokimov, A. L. Perezhogin, "Minimal enumerations of subsets of a finite set and the middle level problem", Discrete Appl. Math., 114:1-3 (2001), 109-114.

[2] C. D. Savage, P. Winkler, "Monotone Gray codes and the middle levels problem", $J$. Combin. Theory Ser. A, 70:2 (1995), 230-248.

[3] I. Shields, C. D. Savage, "A Hamilton path heuristic with applications to the middle two levels problem", Proceedings of the Thirtieth Southeastern International Conference on Combinatorics, Graph Theory, and Computing (Boca Raton, FL, 1999), Congr. Numer., 140, 1999, 161-178. 


\section{К разделу 10}

[1] R. W. Hamming, "Error detecting and error correcting codes", Bell System Tech. J., 29:2 (1950), 147-160; рус. пер.: Р. Хэмминг, "Коды с обнаружением и исправлением ошибок", Коды с обнаружением и исправлением ошибок, ИЛ, М., 1956, $7-22$.

[2] Ю.Л. Васильев, “О негрупповых плотно упакованных кодах", Проблемы кибернетики, № 8, Физматлит, М., 1962, 337-339.

[3] Д. С. Кротов, "Нижние оценки числа $m$-квазигрупп порядка 4 и числа совершенных двоичных кодов", Дискрет. анализ и исслед. опер. Сер. 1, 7:2 (2000), 47-53.

[4] D. S. Krotov, S. V. Avgustinovich, "On the number of 1-perfect binary codes: a lower bound", IEEE Trans. Inform. Theory, 54:4 (2008), 1760-1765.

[5] С.А. Малюгин, "О нижней оценке числа совершенных двоичных кодов", Дискрет. анализ и исслед. опер. Сер. 1, 6:4 (1999), 44-48.

[6] С. В. Августинович, "Об одном свойстве совершенных двоичных кодов”, Дискрет. анализ и исслед. опер., 2:1 (1995), 4-6; англ. пер.: S. V. Avgustinovich, "On a property of perfect binary codes", Operations research and discrete analysis, Math. Appl., 391, Kluwer, Dordrecht, 1997, 13-15.

\section{К разделу 11}

[1] Ю. А. Зуев, "Пороговые функции и пороговые представления булевых функций”, Математические вопросы кибернетики, № 5, Физматлит, М., 1994, 5-61.

[2] E. Goto, H. Takahasi, "Some theorems useful in threshold logic for enumerating Boolean functions", Information Processing, Proceedings of IFIP Congress (Munich, 1962), North-Holland, Amsterdam, 1963, 747-752.

[3] Э. И. Нечипорук, "О синтезе схем из пороговых элементов", Проблемъ кибернетики, № 11, Физматгиз, М., 1964, 49-62.

[4] S. Yajima, T. Ibaraki, "A lower bound of the number of threshold functions", IEEE Trans. Electron. Comput., EC-14:6 (1965), 926-929; pус. пер.: С. Яджима, Е. Ибараки, "Нижняя оценка числа пороговых функций", Кибернет. сб. Нов. сер., 1969, № $6,72-81$.

[5] Ю. А. Зуев, "Асимптотика логарифма числа пороговых функций алгебры логики", Докл. АН СССР, 306:3 (1989), 528-530; англ. пер.: Yu. A. Zuev, "Asymptotics of the logarithm of the number of threshold functions of the algebra of logic", Sov. Math., Dokl., 39:3 (1989), 512-513.

[6] Ю. А. Зуев, "Комбинаторно-вероятностные и геометрические методы в пороговой логике", Дискрет. матем., 3:2 (1991), 47-57; англ. пер.: Yu. A. Zuev, "Methods of geometry and probabilistic combinatorics in threshold logic", Discrete Math. Appl., 2:4 (1992), 427-438.

[7] А.А. Ирматов, "О числе пороговых функций”, Дискрет. матем., 5:3 (1993), 40-43; англ. пер.: А. A. Irmatov, "On the number of threshold functions", Discrete Math. Appl., 3:4 (1993), 429-432.

\section{К разделу 12}

[1] М. Холл, Комбинаторика, Мир, М., 1970; пер. с англ.: M. Hall, Jr., Combinatorial theory, Blaisdell, Waltham-Toronto-London, 1967.

[2] J. Doyen, G. Valette, "On the number of non isomorphic Steiner triple systems", Math. Z., 120:2 (1971), 178-192.

[3] Г. П. Егорычев, “Доказательство гипотезы ван дер Вардена для перманентов", Сиб. матем. журн., 22:6 (1981), 65-71; англ. пер.: G. P. Egorychev, "The solution of van der Waerden's problem for permanents", Adv. Math., 42:3 (1981), 299-305. 


\section{К разделу 13}

[1] J. A. Bondy, "A graph reconstructor's manual", Surveys in combinatorics, Proceedings of the 13th British Combinatorics Conference (Guildford, 1991), London Math. Soc. Lecture Note Ser., 166, Cambridge Univ. Press, Cambridge, 1991, 221-252.

[2] M. Bilinski, Y.S. Kwon, X. Yu, "On reconstruction of planar graphs", J. Combin. Theory. Ser. B, 97:5 (2007), 745-756.

[3] S. Fiorini, J. Lauri, "The reconstruction of maximal planar graphs. I: Recognition", J. Combin. Theory Ser. B, 30:2 (1981), 188-195.

[4] W. B. Giles, "The reconstruction of outerplanar graphs", J. Combin. Theory Ser. B, 16:3 (1974), 215-226.

[5] P. J. Kelly, "A congruence theorem for trees", Pacific J. Math., 7:1 (1957), 961-968.

[6] J. Lauri, "The reconstruction of maximal planar graphs, II: Reconstruction", J. Combin. Theory Ser. B, 30:2 (1981), 196-214.

[7] S. M. Ulam, A collection of mathematical problems, Interscience Tracts Pure Appl. Math., 8, Wiley, New York, 1960; 2nd ed.: Problems in modern mathematics, Wiley, New York, 1964; рус. пер. 1-го изд.: С. Улам, Нерешенные математические задачи, Современные проблемы математики, Наука, М., 1964.

А. Д. Коршунов (A. D. Korshunov)

Институт математики им. С. Л. Соболева СО РАН

E-mail: korshun@math.nsc.ru
Поступила в редакцию

28.08.2009 